Vol. 2, No. 2, 2021

ISSN (ONLINE) 2597-0593

DOI 10.7146/njlis.v2i2.129677

(C) CC BY-NC-ND 4.0

Nanna Kann-Rasmussen, Associate Professor, Department of Communication, University of Copenhagen, Denmark, nkr@hum.ku.dk

\title{
Book review:
}

\section{Atmospheres of surveillance}

Karen Grova Søilen (2021). Atmospheres of surveillance. Københavns Universitet, Det Humanistiske Fakultet.

Atmospheres of surveillance by Karen Grova Søilen is a PhD thesis in information studies. The motivation for the thesis is a need to understand and describe the experience of surveillance in a contemporary setting and with a renewed focus on the body. As such, Søilen situates the thesis within the field of surveillance studies. The overarching research question for the thesis is "in what ways may the concept 'atmospheres of surveillance' contribute to our understanding of the embodied, multisensory experience of contemporary surveillance culture" (p. 18). The thesis answers this question in three stages: a) a theoretical development of the concept "atmospheres of surveillance", b) an empirical reading of three different artworks (all video installation artworks) centered on the theme surveillance and c) a methodological exploration of "atmospheric writing" as a novel way to engage with the theme of the thesis, Atmospheres of Surveillance (henceforth AoS).

The first chapter sets out to build a comprehensive theoretical foundation for developing a vocabulary for AoS and further refine the argument that surveillance contains and co-produces atmospheres. This is done through discussions of the concept of surveillance, which builds up to Søilen's own understanding of the concept as primarily a mode of directing, as a means to influence behavior and produce a repertoire. After this, the chapter goes on to discuss the concept of atmosphere through independent and critical readings of the works of Hermann Schmitz and Gernot Böhme, which Søilen uses to emphasize that atmospheres can be deliberately produced (similar to stage setting), and that production and perception of atmospheres are two equally important aspects in the concept. Through readings primarily of Sara Ahmed, she concludes that atmospheres can be experienced differently depending on the perceiving living body. The chapter closes with an emphasis on the critical potential of the concept AoS.

The second chapter has a two-fold objective. The first is to deal with the methodological problems that arise when describing atmospheres of surveillance in artworks. The second is to analyze the artwork Safe Conduct by Ed Atkins (2016). The chapter addresses the sub-question: What bodily, 
emotional, and sensory experiences of surveillance surface through the prism of contemporary art, and how can 'atmospheres of surveillance' be explored methodologically? Søilen discusses the specific challenges related to the concept of atmospheres with concepts from ethnography, phenomenology, and literary scholarship. She introduces the concept of atmospheric writing as a method that can make an embodied experience of atmospheres of surveillance available to others (p. 80). Atmospheric writing is thus the proposed answer to the methodological problems that Søilen describes. The method is inspired by feminist scholarship, and even answers to the challenge of how to research something that is dependent on lived bodily experience. The last part of the chapter relates to the artwork Safe Conduct. Traditional analysis and atmospheric writing are interweaved throughout the last half of the chapter. Søilen concludes that art opens up new perspectives on how AoS subtly and suggestively penetrate everyday life and that a sensitivity towards AoS can provide a critical space for reflection.

The objective of the third chapter of the thesis is to address the sub-question: How can the notion of 'atmospheres of surveillance' illuminate traces of the violence and power relations of surveillance? Søilen introduces the concept of haunting as a dimension of AoS, which can emphasize the critical potential of her concept because it "may add an additional layer of receptivity to the violence, politics, and power dimensions of surveillance and its historical continuities" (p. 106). Søilen describes the home security/surveillance system "Lighthouse" and connects home surveillance systems to military affordances of domination, and violence. The middle part of the chapter is an analysis of the artwork Modern Escape by the artist duo Hanne Nielsen and Birgit Johnsen (2018). As in chapter two, a traditional analysis of the artwork is interweaved with the personal experience using atmospheric writing. The chapter then discusses the entanglement of (post 9/11) warfare, security, and the private home. This discussion is primarily (though not entirely) informed by theories of aesthetics. Through a discussion of the uncanny, and the dissolving borders of the modern home as well as the etymological connection between "home" and "haunted", Søilen concludes that the modern home becomes "the haunted house" through the introduction of the military-industrial complex in modern surveillance technologies of everyday life.

In addition to the ongoing investigation of how contemporary artworks reflect how surveillance felt, the fourth chapter aims to close a theoretical gap between "the digital" and the concept of atmospheres. Thus, Chapter 4 answers the sub-question: How can 'atmospheres of surveillance' articulate affective and social experiences distinct to the present cultural and historical moment of the early decades of the twenty-first century? Søilen focuses on Raymond Williams' concept "structures of feelings" to capture atmospheres with longer durations of time and more collective feelings. She concludes that theorizing AoS requires attention to the digital because they exist in hybrid spaces, where the physical and the digital is intertwined. The last part of the chapter is an analysis of the artwork Factory of the Sun by German artist Hito Steyerl (2015). The work is analyzed with focus on the feeling of ambient entrapment, which she characterizes as a feeling born out of particularly present-day digital surveillance, algorithmic predictions, and the digital data economy. Another distinct result of the analysis pertains to gamification and the blurred borders between work and play as well as between free and paid for. The chapter ends by demonstrating how the atmospheric writing vignettes have allowed Søilen to communicate a certain feeling of numbness and apathy that is related to the artwork and the AoS it conveys.

The conclusion of the thesis revisits the chapters, the research questions and the sub-questions and clarifies the contribution of the thesis. Søilen thus 1) offers a conceptualization of atmospheres of surveillance by developing a theoretical vocabulary 2) develops a methodology of written vignettes of atmospheric writing and 3) describes how atmospheres of surveillance are articulated in a selection of contemporary artworks.

Kann-Rasmussen: Atmospheres of surveillance (book review) 
The dissertation makes a very strong case for analyzing what surveillance is and what it does from an artistic standpoint, and not from the classic sociological points of view, which have arguably prevailed in surveillance studies until just a few years ago. One of the dissertation's main contributions is to deliver an extremely distinct and original approach to the question: how does contemporary surveillance influence emotions and shape behavior? The artworks are analyzed in the light of appropriate theories of atmosphere drawn from phenomenology and other cultural theories. Furthermore, the analysis is enriched by experimental methodology (the written vignettes from the author's creative writing). In this way, the dissertation makes a very persuasive case for how 'atmospheres of surveillance' can be explored, namely through multiple methodological and experiential viewpoints. The methodological contribution will represent an inspiring example for other scholars working in this field.

Another of the project's key contributions is to show how encountering artworks changes thinking and even changes the way in which atmospheres can be felt. Especially helpful to this, is the way in which the dissertation argues for a need to move beyond the predominance of visual metaphors in surveillance studies in order to encompass multisensory experience. This focus is especially important and makes for an urgently needed contribution to multi-disciplinary surveillance studies. To move away from an optically centered understanding of surveillance is extremely important from feminist and decolonial points of view. Surveillance does no longer only take place through these. It therefore makes very good sense that the dissertation focuses on corporeal experiences of contemporary surveillance culture.

The strong points in the thesis easily overshadow the weaknesses. However, there are a few points that could have been improved.

The dissertation relates to a discussion between David Lyon and James Harding about how to define surveillance (chapter 1). Søilen acknowledges Lyon's general point about surveillance being "Janusfaced", that is ambivalent in the sense that it is both about control and care, and she maintains that surveillance in general is a complex and multifaceted phenomenon. However, Søilen seems to follow Harding's critique of the very idea of positive or good aspects of surveillance, and that such a perspective will somehow diminish proper critical thinking about the dangers of surveillance, especially in the age of digital technologies. This is obviously a legitimate concern, but at the same time Søilen appears to overlook the inherent problem in disregarding scholarship that does not subscribe to this particular "critical" approach to surveillance studies. It could have been fruitful and would have strengthened the concept of "atmospheres of surveillance" to have engaged constructively with a broader body of literature and thus have situated the thesis more thoroughly in surveillance studies.

Methodologically it would have been good to see some more reflections on the author herself in relation to the vignettes of atmospheric writing. Søilen makes a strong argument in chapter 2 for the viewpoint that atmospheres can be experienced differently depending on the perceiving living body and reflects upon this in a hypothetical way regarding surveillance in airports. However, there are no reflections of possible consequences of Søilen's particular perceptions of the artworks, as a critical researcher analyzing critical artworks on the same topic as her research.

Overall, Karen Grova Søilen's PhD dissertation represents an excellent contribution to the so-called 'cultural turn' in the multidisciplinary field of surveillance studies. The dissertation's focus on experiences of surveillance and the emphasis on a non-visual, embodied approach fills a gap in the scholarship and brings valuable insights to the field of surveillance studies. Also, the concept of AoS,

Kann-Rasmussen: Atmospheres of surveillance (book review) 
Nordic Journal of Library and Information Studies

is an original contribution which is shown in the dissertation to be useful by highlighting modes of surveillance which are rarely studied. The method of "atmospheric writing" is an innovative contribution, which adds to the qualitative methods of the field of surveillance studies. 